\title{
Water-in-water droplets by passive microfluidic flow focusing
}

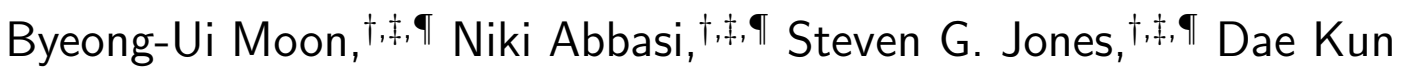

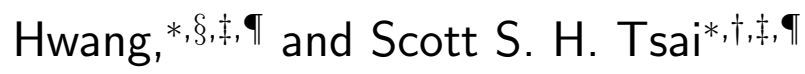 \\ $\dagger$ Department of Mechanical and Industrial Engineering, Ryerson University, Toronto, \\ Canada \\ $\ddagger$ Keenan Research Centre for Biomedical Science, St. Michael's Hospital, Toronto, Canada \\ 9Institute for Biomedical Engineering, Science and Technology (iBEST), a partnership \\ between Ryerson University and St. Michael's Hospital \\ $\S$ Department of Chemical Engineering, Ryerson University, Toronto, Canada \\ E-mail: dkhwang@ryerson.ca; scott.tsai@ryerson.ca
}

\begin{abstract}
We present a simple microfluidic system that generates water-in-water, aqueous two phase system (ATPS) droplets, by passive flow focusing. ATPS droplet formation is achieved by applying weak hydrostatic pressures, with liquid-filled pipette tips as fluid columns at the inlets, to introduce low speed flows to the flow focusing junction. To control the size of the droplets, we systematically vary the interfacial tension and viscosity of the ATPS fluids, and adjust the fluid column height at the fluid inlets. The size of the droplets scales with a power-law of the ratio of viscous stresses in the two ATPS phases. Overall, we find a drop size coefficient of variation (CV; i.e. polydispersity) of about $10 \%$. We also find that when drops form very close to the flow focusing junction, the drops have CV of less than $1 \%$. Our droplet generation method is easily
\end{abstract}


scalable: we demonstrate a parallel system that generates droplets simultaneously, and improves the droplet production rate by up to one order-of-magnitude. Finally, we show the potential application of our system for encapsulating cells in water-in-water emulsions, by encapsulating microparticles and cells. To the best of our knowledge, our microfluidic technique is the first that forms low interfacial tension ATPS droplets without applying external perturbations. We anticipate that this simple approach will find utility in drug and cell delivery applications because of the all-biocompatible nature of the water-in-water ATPS environment.

\section{Introduction}

An aqueous two-phase system (ATPS) is formed when two incompatible polymers, such as polyethylene glycol (PEG) and dextran (DEX), are mixed in water and phase-separated above a critical polymer concentration. ${ }^{1}$ The biocompatible nature of ATPS has resulted in their application in several biomedical settings, such as in DNA extraction, ${ }^{2}$ protein partitioning, ${ }^{3}$ and cell separation. ${ }^{4}$

A distinguishing feature of ATPS is that the interfacial tension between different liquid phases tends to be very low-often less than $10^{-4} \mathrm{~N} / \mathrm{m} .{ }^{5}$ In microfluidics, such low interfacial tensions make generating droplets very challenging. For example, while microfluidic flow focusing has often been used to create water-in-oil or oil-in-water droplets, ${ }^{6,7}$ flow focusing ATPS fluids has resulted in very long threads that either survive indefinitely in the microchannel, or breakup erratically far downstream of the focusing junction. ${ }^{8,9}$ So far, the only practical ways of producing ATPS droplets in microfluidics have required external forcing such as electrohydrodynamic perturbation, ${ }^{10,11}$ oscillating piezoelectric disks, ${ }^{12,13}$ pin actuation, ${ }^{14}$ mechanical vibration, ${ }^{15-17}$ and pulsating inlet pressure. ${ }^{18}$

All of these systems require specialized external components that actively perturb the fluids at a controlled frequency. The externally actuating components often involve com-

plex customization to setup. Therefore, despite the promise of all-biocompatibility, these 
challenges have prohibited the wide-spread use of ATPS in droplet-based microfluidics, and make a simple and accessible ATPS droplet generation technique very desirable. ${ }^{19}$

Here, we present a simple microfluidic approach to passively make ATPS droplets. Our technique exploits gravity-driven hydrostatic flow in a flow focusing geometry. The disperse DEX and continuous PEG phases are injected through the microfluidic inlets by vertically inserted and solution-filled pipette tips. We control the very low speed flow conditions, by modulating the hydrostatic fluid column heights in the pipettes. Importantly, our setup does not require any external components such as pumps or oscillating elements, making our technique very easy to implement.

In experiments, we systematically vary the interfacial tension and viscosity of the ATPS, and the column heights of DEX and PEG in the pipette tips, to define droplet formation regimes and measure drop sizes. We find that the resulting droplet sizes scale with a powerlaw of the ratio of the viscous stresses in the two phases. To demonstrate easy scale-up of our passive ATPS droplet formation method, we design a multiple droplet generator and perform experiments to create four drops simultaneously in four parallel channels. Finally, we demonstrate the proof-of-concept encapsulation application of our passive ATPS droplet generator, by encapsulating microparticles and cells.

\section{Experimental section}

\section{Chemicals}

We prepare ATPS solutions by combining two mixtures of $5 \%(\mathrm{w} / \mathrm{v})$ or $10 \%(\mathrm{w} / \mathrm{v})$ PEG (Mw 35k, Sigma-Aldrich, St. Louis, MI, USA) and $16 \%$ (w/v) DEX (Mw 500k, Pharmacosmos, Holbaek, Denmark). Each pair of $100 \mathrm{~mL}$ stock solutions is made by dissolving the PEG and DEX in deionized (DI) water. The solutions are vigorously mixed in a T25 flask (Thermo

Scientific, Waltham, MA, USA), and allowed to phase-separate for more than 24 hours. Due to the density difference of DEX and PEG, phase-separation leads to a system that has an 
upper PEG-rich phase and a lower DEX-rich phase. We refer the phase-separated solutions of the initially $5 \%(\mathrm{w} / \mathrm{v})$ PEG and $16 \%(\mathrm{w} / \mathrm{v})$ DEX, and $10 \%(\mathrm{w} / \mathrm{v})$ PEG and $16 \%(\mathrm{w} / \mathrm{v})$ DEX, as ATPS 1 and ATPS 2, respectively.

After separation, we use syringes (BD Medical, Franklin Lakes, NJ, USA) to extract the PEG-rich and DEX-rich phases. We measure the viscosities of the solutions with a glass viscometer. The viscosities of the ATPS 1 PEG-rich and DEX-rich solutions are $\mu_{P}=9.9$ $\mathrm{mPa} \mathrm{s}$ and $\mu_{D}=38.0 \mathrm{mPa}$ s, respectively. Viscosities of the ATPS 2 PEG-rich and DEXrich solutions are $\mu_{P}=17.0 \mathrm{mPa} \mathrm{s}$ and $\mu_{D}=71.9 \mathrm{mPa}$ s, respectively. From the reported measurements of Atefi et al. ${ }^{20}$ we find that ATPS 1 and 2 have ultralow interfacial tensions, $\gamma=0.037$ and $0.103 \mathrm{mN} / \mathrm{m}$, respectively.

\section{Microparticles and cells}

In magnetic microparticle encapsulation experiments, we use $10 \mu \mathrm{m}$ diameter polystyrenebased paramagnetic microparticles (Sigma-Aldrich, St. Louis, MI, USA). We mix $100 \mu \mathrm{L}$ of the microparticle stock solution (5\% solid concentration) into $2 \mathrm{~mL}$ of our DEX solution. We also position a stack of two neodymium iron boron (NdFeB) magnets (K. J. Magnetics, Jamison, PA, USA), each with dimensions $12.70 \times 6.35 \times 3.18 \mathrm{~mm}$, adjacent to the microchannel to separate the particle encapsulating droplets.

For cell encapsulation experiments, frozen peripheral blood mononuclear cells (PBMCs; STEMCELL Technologies Inc., Vancouver, Canada) are thawed in a warm water bath and cultured in Dulbeccos modified eagles medium (DMEM) with $10 \%$ fetal bovine serum (FBS). Cells are then incubated at $37^{\circ} \mathrm{C}$ with $5 \% \mathrm{CO}_{2}$ for 24 hours. We magnetically separate the cells using the EasySep ${ }^{\mathrm{TM}}$ Human CD45 Depletion Kit (STEMCELL Technologies Inc., Vancouver, Canada). The separated cells are then suspended in a DEX solution for microfluidic cell encapsulation experiments. 


\section{Device fabrication}

Our microfluidic devices are fabricated by the standard soft lithography method. ${ }^{21} \mathrm{Mi}$ crochannels are drawn with computer-aided design (CAD) software (AutoCAD 2011, Autodesk, Inc., San Rafael, CA, USA), and printed at a high resolution (25,400 dpi; CAD/ART Services Inc., Bandon, OR, USA) on a transparency sheet, to complete a patterned photomask.

A 4-inch diameter silicon wafer serves as the platform for channel feature construction. We spin-coat SU-8 2035 photoresist (Microchem, Newton, MA, USA) on the wafer and expose the wafer to UV light through the patterned photomask. The unexposed photoresist is removed by dissolving the wafer in a developer solution, so that only microchannel features remain on the silicon master.

We prepare a 10:1 ratio mixture of polydimethylsiloxane (PDMS) resin to curing agent (Sylgard 184, Dow Corning, Midland, MI, USA), pour the mixture onto the silicon master, and cure in an oven for 2 hours. After removal of the cured PDMS from the silicon master, we make inlet and outlet holes on the PDMS layer using a $1 \mathrm{~mm}$ diameter biopsy punch (Integra Miltex, Inc., Rietheim-Weilheim, Germany). These holes are used to vertically insert the pipette tips. After cleaning the PDMS layer and a glass cover slide, we bond the PDMS to the glass slide irreversibly using oxygen plasma treatment (Harrick Plasma, Ithaca, NY, USA). For the two-layer microfluidic device, used to scale-up production of ATPS droplets,

we bond an additional PDMS sheet on top of the existing PDMS layer. We are careful to ensure proper alignment of the through-holes of the intermediate PDMS sheet.

\section{Experiment setup}

Fig. 1 (a) shows an image of the microfluidic device and a schematic diagram of the flow focusing junction. We generate water-in-water droplets by incorporating weak hydrostatic pressures to drive the flows into the microfluidic channel. $250 \mu \mathrm{L}$ pipette tips that are prefilled with DEX and PEG solutions, are vertically inserted into the inlets of the microfluidic 


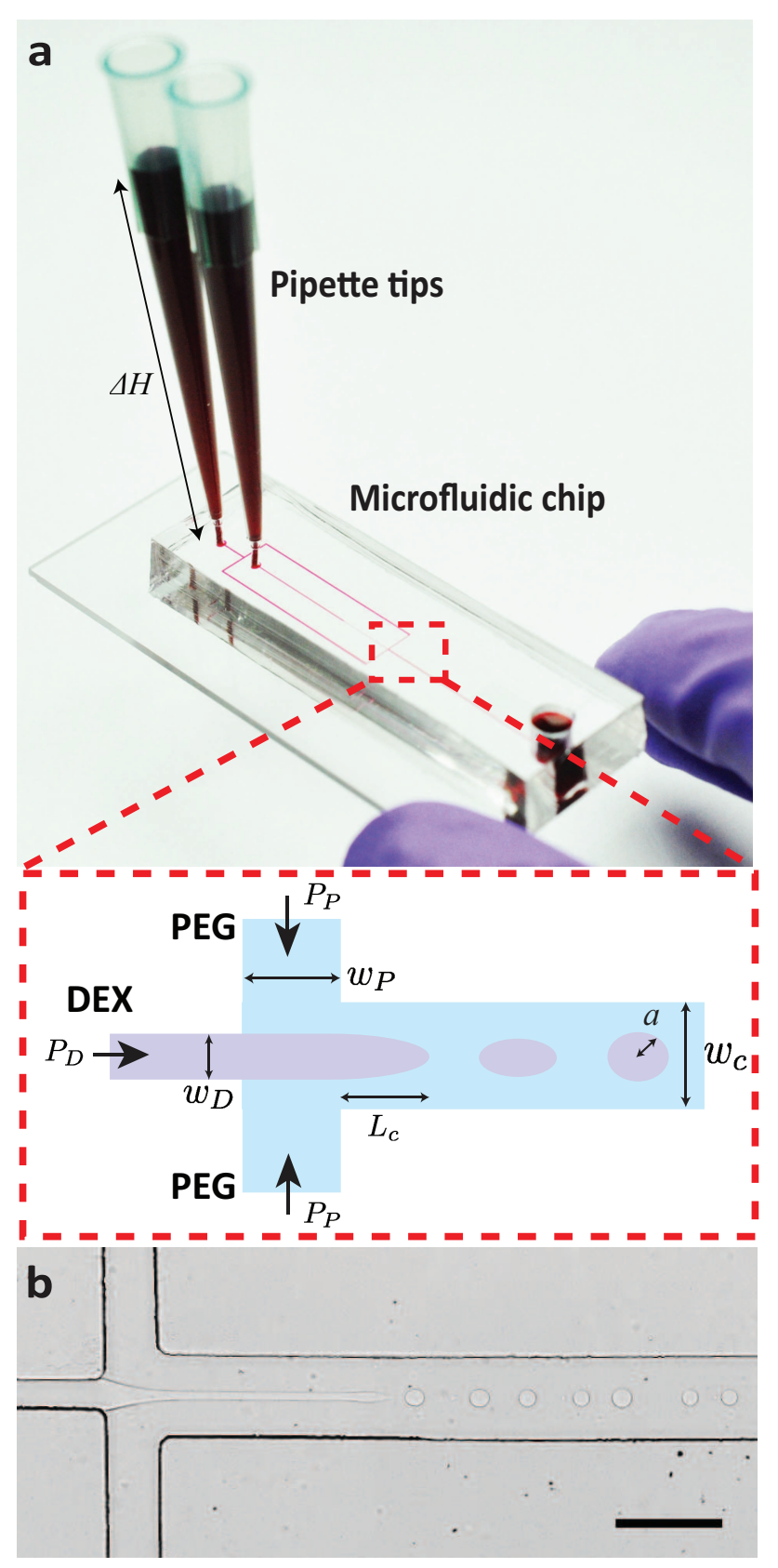

Figure 1: (a) An image of the microfluidic device, and an enlarged schematic diagram of passive ATPS droplet formation. The two pipette tips enable control of gravity-driven hydrostatic flow in the microfluidic channel. The DEX solution is introduced as the inner stream, sheathed into a slender thread by the outer PEG phase, and breaks-up into droplets downstream of the flow-focusing junction. (b) A representative experimental image of ATPS droplet formation in a microfluidic channel. The very low flow rates of the two ATPS phases, enabled by weak hydrostatic pressures at the inlets, achieve droplet formation by a RayleighPlateau instability. In this image, ATPS 1 is used for the DEX and PEG phases, the inner DEX solution is introduced at an inlet pressure $P_{D}=0.41 \mathrm{kPa}$, and the outer PEX phase has an inlet pressure $P_{P}=0.54 \mathrm{kPa}$. Scale bar $200 \mu \mathrm{m}$. 
device.

The DEX and PEG solutions form disperse and continuous phases, respectively. The DEX, PEG, and downstream channels have dimensions $w_{D}=50 \mu \mathrm{m}, w_{P}=100 \mu \mathrm{m}$, and $w_{c}$ $=150 \mu \mathrm{m}$, respectively. All channels have height, $h=50 \mu \mathrm{m}$.

We control the applied hydrostatic pressure by changing the fluid column height, $\Delta H$, in the pipette tips. The column height, $\Delta H$, is measured from the liquid level in the pipette tip to the top of the PDMS sheet. We estimate the microfluidic inlet hydrostatic pressure $P=\rho g \Delta H$, where $\rho$ is the density of the fluid, and $g=9.81 \mathrm{~m} / \mathrm{s}^{2}$ is the acceleration due to gravity.

Experimental images are captured using an inverted microscope (IX71, Olympus Corp., Tokyo, Japan) with 10x or 20x objectives, and an attached high speed camera (Vision Research, Wayne, NJ, USA). The high speed camera is set at a frame rate of $500 \mathrm{fps}$ and an

exposure time of $1 \mathrm{~ms}$, unless otherwise stated. We use Image ${ }^{\mathrm{TM}}$ software to post-process the images, and measure the size of the droplets.

\section{Results and discussion}

\section{ATPS droplets by passive microfluidic flow focusing}

In microfluidic flows that rely on constant flow-rate syringe pumps to drive the fluids, generation of droplets have required application of external forces that perturb the ATPS interface. Breakup of the slender inner thread of the disperse phase by the Rayleigh-Plateau instability alone has not been able to consistently make ATPS drops. ${ }^{19}$ However, in our setup, the slow flow enabled by weak inlet hydrostatic pressures permits droplet formation near the flow focusing junction. To better understand why our system enables passive ATPS thread breakup-when systems that use constant flow-rate syringe pumps do not permit ATPS drop formation-we conduct experiments with both hydrostatic pressure, and constant flow-rate syringe pumps, and compare the results with previously described models of microfluidic 
Rayleigh-Plateau instability. ${ }^{8,22}$

Fig. 1 (b) shows a representative experimental image of water-in-water droplet generation by hydrostatic pressure-based, passive microfluidic flow focusing, using the ATPS 1 mixture. As the inner DEX phase solution enters the flow focusing junction, the outer PEG phase flow focuses the DEX stream, so that a narrow DEX thread is formed. The thread then breaks-up via a Rayleigh-Plateau instability several thread diameters away from the junction (see also Supplementary Information Movie 1). Here, the DEX and PEG phase inlet pressures $P_{D}$ $=0.41 \mathrm{kPa}$ and $P_{P}=0.54 \mathrm{kPa}$, respectively, and the resulting thread has an unperturbed radius $r_{o} \approx 8 \mu \mathrm{m}$.

Passive microfluidic thread breakup by the Rayleigh-Plateau instability has been described previously. ${ }^{8,22}$ Here, we adapt the existing model for our ATPS system, such that the instability growth rate,

$$
\omega=\frac{1}{8} \frac{\gamma}{\mu_{P} h} \frac{F(x, \lambda)\left(k^{2}-k^{4}\right)}{x^{9}\left(1-\lambda^{-1}\right)-x^{5}}
$$

where the dimensionless thread radius $x=r_{o} /(h / 2)$, the viscosity ratio $\lambda=\mu_{D} / \mu_{P}$, the perturbation wavenumber is $k$, and the function,

$$
\begin{array}{r}
F(x, \lambda)=x^{4}\left(4-\lambda^{-1}+4 \ln x\right)+x^{6}\left(-8+4 \lambda^{-1}\right) \\
+x^{8}\left(4-3 \lambda^{-1}-\left(4-4 \lambda^{-1}\right) \ln x\right) .
\end{array}
$$

With a maximum wavenumber, $k=0.7$, we find that ATPS 1 has the Rayleigh-Plateau instability growth rate, $\omega \approx 11 s^{-1}$. 
As in Geschiere et al., ${ }^{8}$ we assume that an initial perturbation of amplitude $\epsilon_{o}=O(1)$ $\mathrm{nm}$, at the flow focusing junction, perturbs the thread radius as $r=r_{o}+\epsilon_{o} e^{\mathrm{i}\left(k / r_{o}\right) z+\omega t}$, where the time $t$ corresponds to the duration of the perturbation growth. We assume that the perturbation grows until $r_{o}=\epsilon_{o} e^{\omega t}$, when the thread ruptures, and we find the thread breakup time $t=\omega^{-1} \ln \left(r_{o} / \epsilon_{o}\right) \approx 0.8 \mathrm{~s}$ for ATPS 1. In Fig. 1 (b), we also use ATPS 1 fluids, and the record thread breakup time $t \approx 1.1 \mathrm{~s}$. This value is in good agreement with the theoretical ATPS 1 thread breakup time $t \approx 0.8 \mathrm{~s}$.

Here, we note that Geschiere et $a l{ }^{8}$ described the perturbation growth rate of their experimental DEX-PEG ATPS thread as being more than one order-of-magnitude lower compared to the theoretical predictions. They ruled out the effects of viscoelasticity of the ATPS fluids, and adhesion to the microchannel walls, as possible contributors to the stability of the ATPS interface. However, they did not arrive at a conclusive explanation for their observations.

Interestingly, in our experiments we do not observe the unexpected stability of ATPS that was found by Geschiere et al. ${ }^{8}$ Therefore, a more systematic study may be necessary to fully understand the relationship between the theoretical model of Rayleigh-Plateau instability, and ATPS droplet breakup experiments.

We observe that, beyond a critical distance $L_{c} \approx 1.7 \mathrm{~mm}$, downstream from the flow focusing junction (Fig. 1 (a)), breakup of the ATPS thread becomes erratic, and the drops formed vary dramatically in size. Therefore, we deem it impractical to have ATPS drops formed beyond the distance $L_{c} \approx 1.7 \mathrm{~mm}$, downstream from the flow focusing junction.

With ATPS 1 fluids, downstream channel cross section $w_{c} \mathrm{x} h=7,500 \mu \mathrm{m}^{2}$, DEX phase thread initial radius $r_{o} \approx 8 \mu \mathrm{m}$, typical thread breakup time $t \approx 0.8 \mathrm{~s}$, and critical breakup distance $L_{c} \approx 1.7 \mathrm{~mm}$, we estimate that passive ATPS droplet formation may be practical up to a maximum channel flow-rate $Q_{c}=w_{c} h\left(L_{c} / t\right) \approx 0.95 \mu \mathrm{L} / \mathrm{min}$. This flow-rate is near the lower limit of most commercially available syringe pumps. Additionally, constant flowrate syringe pumps are known to be unstable near and below $O(1) \mu \mathrm{L} / \mathrm{min},{ }^{23}$ so passively 
generating ATPS droplets with constant flow-rate syringe pumps is not practical.

Indeed, when we attempt to form ATPS droplets using constant flow-rate syringe pumps, we are unable to achieve droplet formation near the flow focusing junction, using the same ATPS 1 mixture. Our Harvard Apparatus Pump 11 Elite has a minimum flow rate of 0.05 $\mu \mathrm{L} / \mathrm{min}$, using a $1 \mathrm{~mL}$ syringe. To find the optimal condition for ATPS droplet generation using constant flow-rate syringe pumps, we apply the minimum flow rate, $0.05 \mu \mathrm{L} / \mathrm{min}$, to the DEX phase, and then systematically tune the PEG phase flow rate until the DEX thread radius $r_{o} \approx 8 \mu \mathrm{m}$ (the value of thread radius $r_{o}$ in our ATPS 1 hydrostatic flow experiments; see Supplementary Information Fig. S1). The corresponding PEG phase flow rate is 1.5 $\mu \mathrm{L} / \mathrm{min}$, so the total flow rate of the system is $1.55 \mu \mathrm{L} / \mathrm{min}$, which is larger than the critical flow rate $Q_{c} \approx 0.95 \mu \mathrm{L} / \mathrm{min}$.

Supplementary Information Fig. S2 shows an experiment with ATPS 1, using constant flow-rate syringe pumps operating at approximately the optimal condition corresponding to DEX thread radius $r_{o} \approx 8 \mu \mathrm{m}$. Despite operating the Pump 11 Elite syringe pump at the absolute minimum flow rate for the DEX phase (as specified by the manufacturer, Harvard Apparatus), the DEX thread still does not break up until very far $(\sim 20 \mathrm{~mm})$ downstream of the flow focusing junction.

When we introduce the same ATPS 1 system using hydrostatic pressure, we are able to obtain a smooth and consistent flow, with a total flow rate of approximately $0.2 \mu \mathrm{L} / \mathrm{min}$ (see Fig. 1 (b)). The flow rate achieved by the hydrostatic pressure driven flow here is one order-of-magnitude lower than that of the syringe-pump driven flow, and more critically, below the threshold flow rate $Q_{c} \approx 0.95 \mu \mathrm{L} / \mathrm{min}$. As a result, ATPS drops are able to form near the flow focusing junction.

We also systematically adjust the PEG and DEX pressures, to define a specific regime where ATPS droplet generation is possible in our hydrostatic driven flow setup. Figs. 2 (a) and (b) show the results of the phase diagram. We observe either long slender DEX threads (empty triangles), droplet formation (solid diamonds), or DEX phase back flow (crosses). 

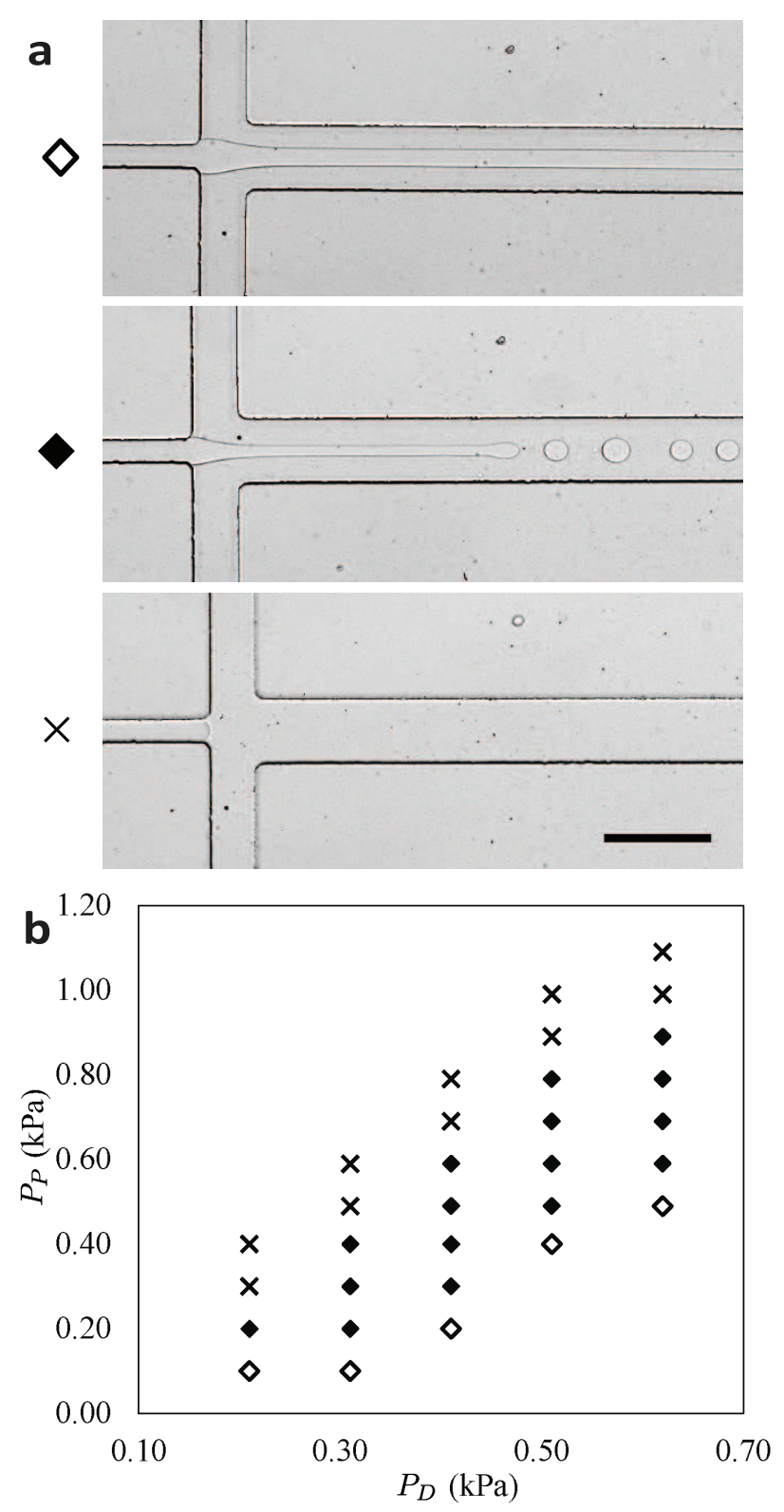

Figure 2: We systematically tune the DEX and PEG phase inlet pressures, by adjusting the fluid column heights of the pipette tips. (a) We find instances of long thread formation (empty diamonds), ATPS droplet generation (solid diamonds), and DEX phase back flow (crosses). (b) The three distinct regimes are indicated on a phase diagram. Scale bar 200 $\mu \mathrm{m}$. 
We note that, when the DEX phase inlet pressure is much higher than the PEG phase inlet pressure, $P_{D}>>P_{P}$, the DEX phase forms a thread that does not break up into droplets within our critical distance $L_{c}$ (empty diamonds in Fig. 2). When the DEX and PEG phase inlet pressures are comparable, $P_{D} \approx P_{P}$, the DEX phase flow is sufficiently slow to cause the DEX thread to breakup into droplets (solid diamonds in Fig. 2). The droplets rapidly become spheres as they leave the jetting tip. At even lower values of the DEX to PEG phase pressure ratio, where $P_{D}<<P_{P}$, the DEX phase back flows due to the much higher pressure of the PEG phase. As a result, neither threads or droplets form in this regime (crosses in Fig. 2).

\section{Controlling ATPS droplet size}

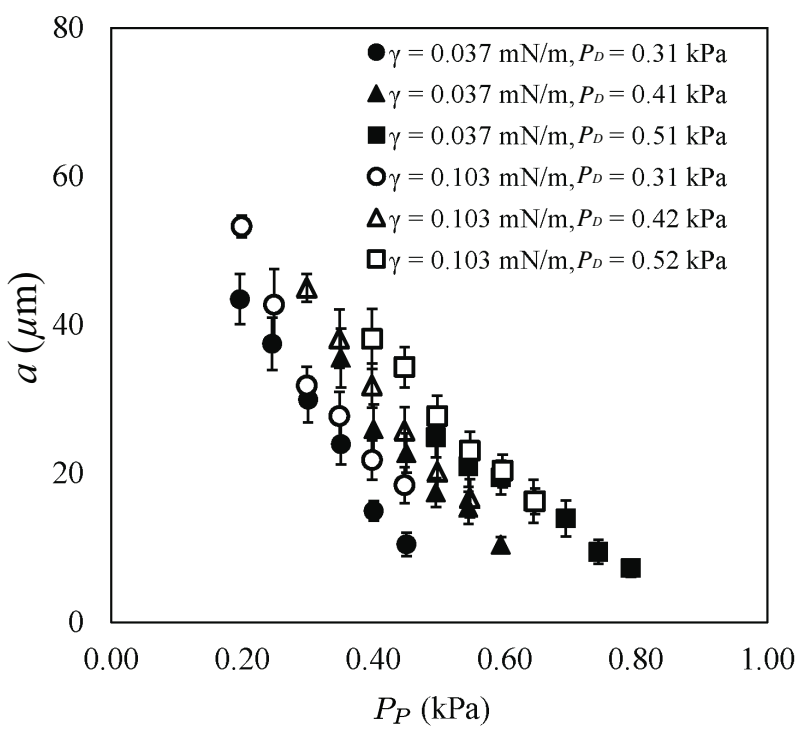

Figure 3: A plot of the measured DEX phase droplet radius $a$, versus the applied PEG phase inlet pressure $P_{P}$. Here, the results are from two different ATPS solutions, ATPS 1 and 2 , with interfacial tensions, $\gamma=0.037$ and $0.103 \mathrm{mN} / \mathrm{m}$, respectively.

We measure the size of droplets generated by our passive flow focusing system, to study how the applied pressures can be adjusted to control drop sizes. Two different types of ATPS solutions are used, so that we can obtain data that reflect two interfacial tensions, and two sets of DEX and PEG viscosities. 
Fig. 3 shows a plot of the measured DEX phase droplet radius $a$, versus the applied PEG phase inlet pressure $P_{P}$. The interfacial tensions for ATPS 1 and 2 are $\gamma=0.037$ and 0.103 $\mathrm{mN} / \mathrm{m}$, respectively. We apply three different DEX phase inlet pressures in experiment with ATPS 1 and 2 (see legend of Fig. 3).

In every set of experiments, we observe a monotonic decrease of the droplet radius as the PEG phase pressure increases. Generally, ATPS 2 (which has a higher interfacial tension) exhibits marginally higher drop sizes compared to ATPS 1 . However, the differences in ATPS 1 and 2's interfacial tension and viscosities do not appear to contribute significantly to the resulting droplet sizes.

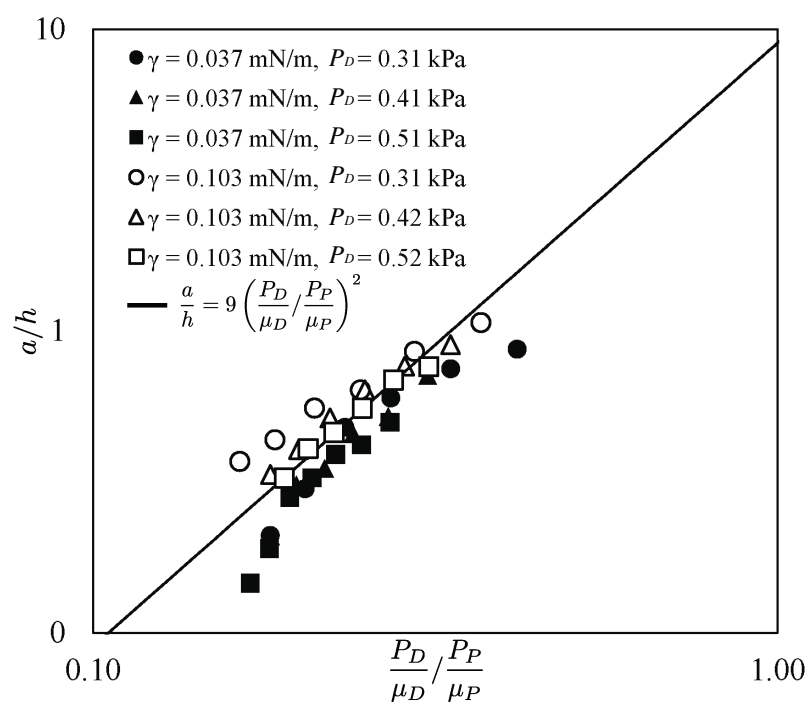

Figure 4: $\log -\log$ plot of dimensionless ATPS droplet diameter $a / h$ versus the ratio of viscous stresses $\mu_{P} P_{D} / \mu_{D} P_{P}$. Our data appears to fit reasonably onto the line indicated by $a / h=9\left(\frac{P_{D}}{\mu_{D}} / \frac{P_{P}}{\mu_{P}}\right)^{2}$.

To obtain an empirical relationship between the resulting ATPS drop size and the controllable inlet conditions, we plot on a $\log$-log graph, the dimensionless drop size $a / h$, against a dimensionless ratio of DEX and PEG phase inlet conditions, $\frac{P_{D}}{\mu_{D}} / \frac{P_{P}}{\mu_{P}}$ (Fig. 4). Our ATPS droplet size data appears to have a reasonable fit with the power-law relationship, $a / h \propto\left(\frac{P_{D}}{\mu_{D}} / \frac{P_{P}}{\mu_{P}}\right)^{2}$. Interestingly, this empirical power-law relationship also appeared in pressure-driven microfluidic droplet generators that used oil-water mixtures. ${ }^{24}$ 
We note that, generally, the polydispersity of our droplets' size is higher than those reported in microfluidic water-oil emulsion generation schemes. ${ }^{25,26}$ Our ATPS 1 and 2 droplet diameters have average coefficients of variance (CV) 12.5 and $10.5 \%$, respectively (averaged from 15 samples of every combination of PEG and DEX phase inlet pressure). Due to the ultralow interfacial tension of ATPS, most of our ATPS droplet generation occurs in the "jetting" regime, relying on Rayleigh-Plateau instability breakup of the central DEX thread. This is in contrast to many microfluidic water-oil emulsion generation setups where droplets are formed in the "dripping regime", which results in much more monodisperse droplets. ${ }^{27}$

To achieve more monodisperse droplets, we reduce the PEG and DEX phase inlet pressures to $P_{P}=0.20$ and $P_{D}=0.31 \mathrm{kPa}$, respectively, and use the higher interfacial tension ATPS 2 mixture. At these very low applied pressures, ATPS droplets form near the flow focusing junction (see Supplementary Information Fig. S3 and Movie 2), in a "dripping" like fashion. The resulting ATPS droplets are highly monodisperse, with CV less than 1 $\%$ (averaged from 53 samples). This result suggests that our passive ATPS droplet generation scheme may also be applied to generate monodisperse droplets - albeit at low applied pressures with a reduced production rate.

\section{Scaled-up production with four parallel orifices}

Our passive droplet formation method uses weak hydrostatic pressures from the fluid columns in pipette tips to control the very low speed flows in our microchannels. While the very low speed flow enables ATPS droplet formation via the Rayleigh-Plateau instability, the slow flow also results in reduced droplet production rates: our microfluidic technique typically

generates ATPS droplets at $O(1)$ drops/sec (which is comparable with some other ATPS droplet generation approaches $\left.{ }^{14,16}\right)$.

Nevertheless, with hydrostatic pressure driving the fluid flow, we are able to easily increase the production rate by multiplexing the process on a single microfluidic chip. Fig. 5 (a) shows a schematic diagram of our multiplexed ATPS droplet generator. Two layers of 


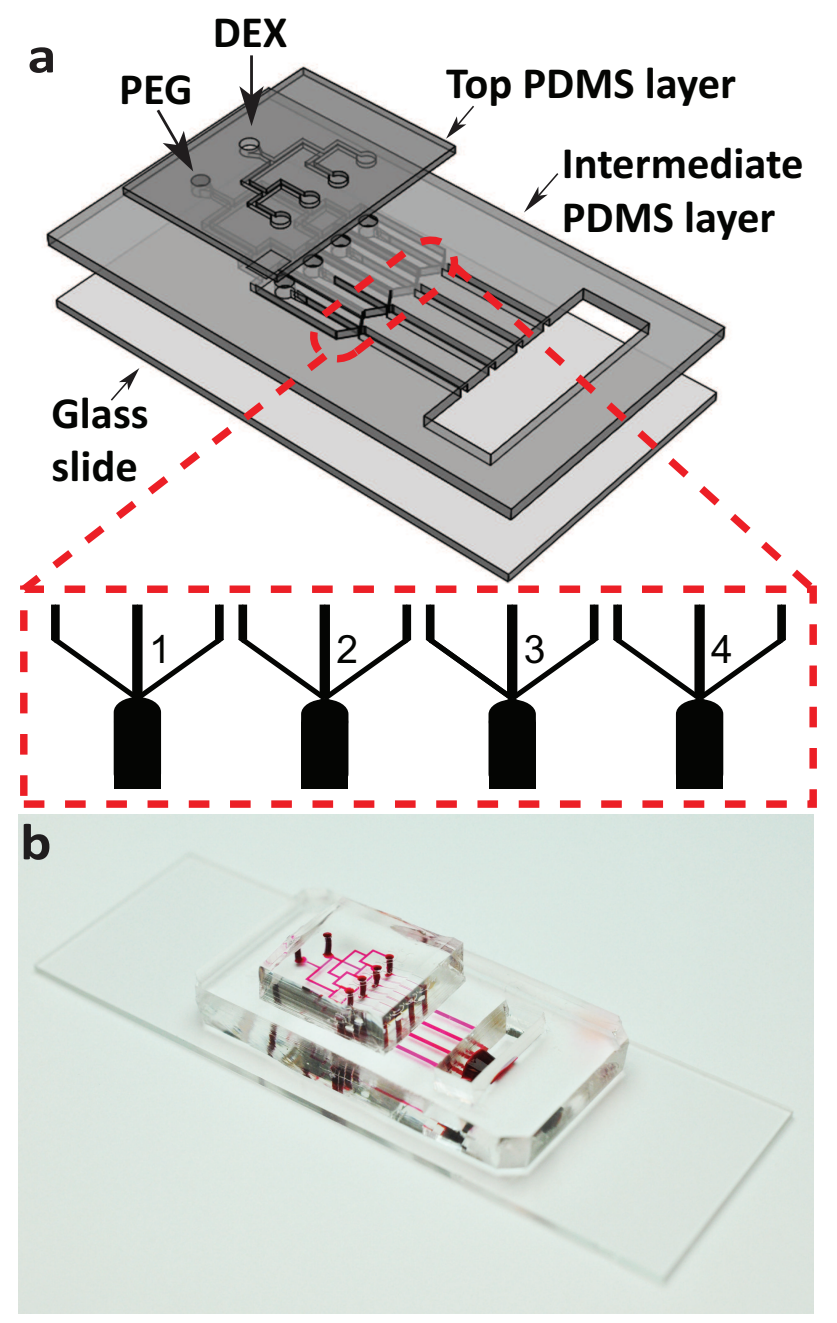

Figure 5: (a) Schematic diagram of a multiple droplet generator. Droplets simultaneously form in an integrated system of four parallel flow focusing junctions. The DEX phase enters via an inlet on the top PDMS layer, and the PEG phase enters via an inlet on the intermediate PDMS sheet. The four flow focusing junctions are identical. (b) An image of the bonded two-layer PDMS-glass device, with colored dye in the microchannels. 
a

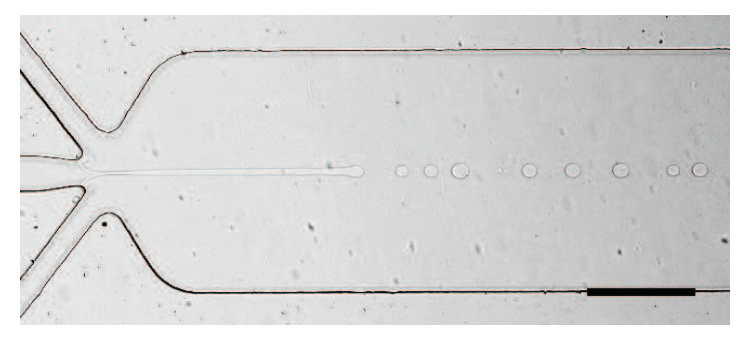

b
Orifice 1

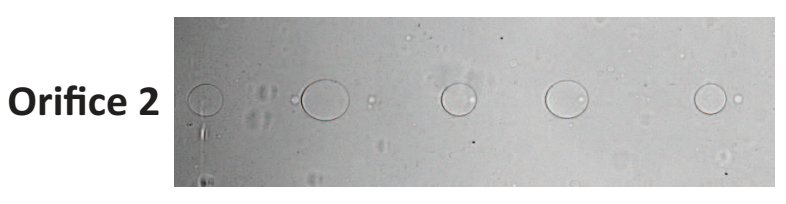

Orifice 4

\subsubsection{0}

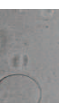

3

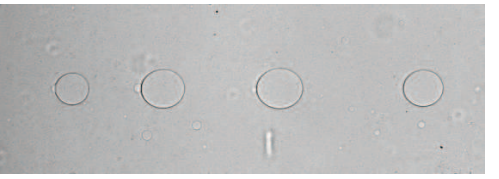

Orifice 3
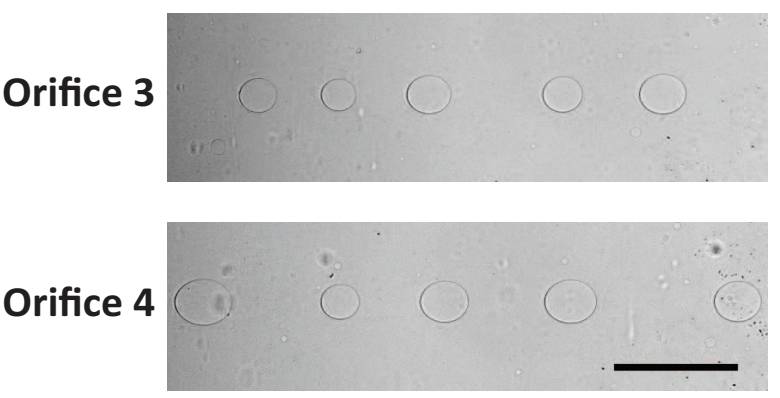
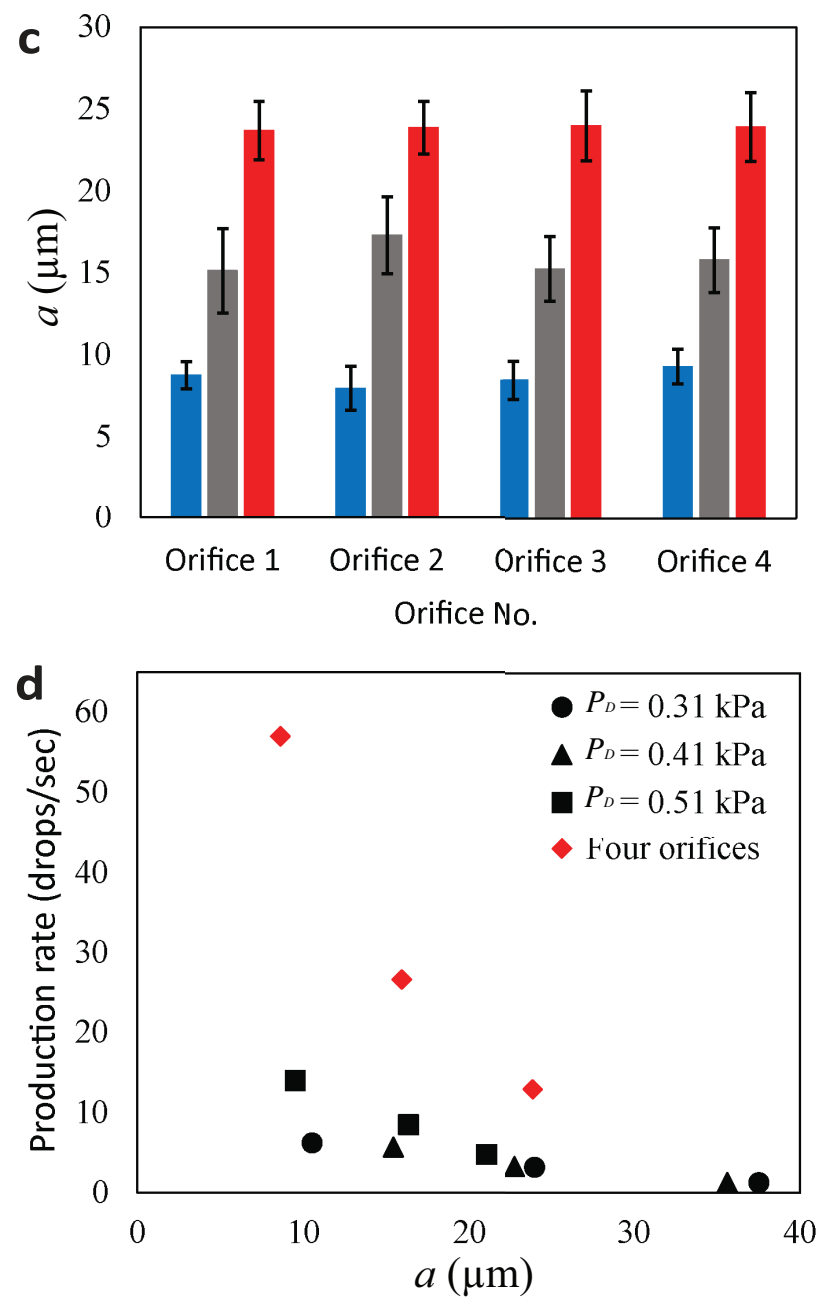

Figure 6: (a) An experimental image of one of the four parallel flow focusing junctions. PEG and DEX phases are introduced at inlet pressures $P_{P}=2.57 \mathrm{kPa}$ and $P_{D}=0.10$ $\mathrm{kPa}$, respectively. (b) Images of the microchannels downstream of the four parallel orifices, showing simultaneous ATPS droplet generation. Here, the PEG and DEX phase inlet pressures $P_{P}=1.98 \mathrm{kPa}$ and $P_{D}=0.31 \mathrm{kPa}$, respectively. Scale bar $200 \mu \mathrm{m}$. (c) A bar graph of ATPS drop radius $a$ versus orifice number. The difference in drop size across the four parallel orifices is insignificant. Here, DEX phase inlet pressure $P_{D}=0.31 \mathrm{kPa}$ is constant. Blue, gray, and red bars represent PEG phase inlet pressures $P_{P}=2.57,2.28$, and $1.98 \mathrm{kPa}$, respectively. (d) A comparison of ATPS droplet production rate versus drop radius $a$ shows dramatic increase in production rate with the multiplexed system. 
PDMS layers are bonded together, and then the bottom PDMS sheet is bonded against a glass slide. The DEX phase enters via the inlet of the top PDMS layer, and is evenly distributed to four branched channels. The PEG phase enters from the inlet of the intermediate PDMS layer, and distributes to eight downstream microchannels. The top and intermediate PDMS sheets are connected via through-holes. All inlets and through-holes have $1 \mathrm{~mm}$ diameter. As shown in the expanded portion of Fig. 5 (a), the device achieves four ATPS droplet generating flow focusing orifices. Each orifice has $40 \mu \mathrm{m}$ width. Fig. 5 (b) shows an image of the fabricated two-layer PDMS-glass bonded device, with microchannels filled with red dye to improve visualization. We note that in this multiplexed system, the DEX and PEG phase fluids are still each controlled from a single pipette tip.

In Fig. 6, we study the multiplexed passive generation of ATPS droplets. We use essentially the same experimental methods as in the single junction system described previously. However, due to the higher overall flow resistance in the microchannels of the multiplexed setup, we use Tygon tubing to achieve a higher PEG phase fluid column. The DEX solution is still supplied via a single pipette tip at the DEX inlet.

Fig. 6 (a) shows a representative image of ATPS droplet generation from one of the four orifices. In a similar fashion to the single junction setup, we observe the thinning and breakup of the DEX thread into individual ATPS droplets. In this image, the continuous PEG phase and discontinuous DEX phase are supplied at inlet pressures, $P_{P}=2.57 \mathrm{kPa}$ and $P_{D}=0.10 \mathrm{kPa}$, respectively, using the ATPS 1 mixture. Fig. 6 (b) shows the resulting ATPS drops downstream of the four orifices. Here, PEG and DEX solutions are introduced at inlet pressures, $P_{P}=1.98 \mathrm{kPa}$ and $P_{D}=0.31 \mathrm{kPa}$, respectively (see also Supplementary Information Movie 3).

We measure the radius of the ATPS droplets as we vary the inlet pressure conditions of the two phases in our multiplexed setup. As shown in the bar graph of Fig. 6 (c), droplet sizes remain consistent despite generation from four different orifices. Here, blue, gray, and red bars represent PEG inlet pressures $P_{P}=2.57,2.28$, and $1.98 \mathrm{kPa}$, respectively, while 
the DEX phase inlet pressure $P_{D}=0.31 \mathrm{kPa}$.

Fig. 6 (d) shows a comparison of the ATPS droplet production rate versus droplet radius a. The multiplexed system, with four ATPS droplet generating orifices, is able to achieve up to $50+$ drops/sec, which is an approximately one order-of-magnitude improvement over the single junction system. This proof-of-concept demonstration suggests that it would not be difficult to achieve an even higher level of scale-up, by simply increasing the number of ATPS droplet generating orifices in our setup. Importantly, due to pressure-driven flow, only one PEG inlet and one DEX inlet are required, regardless of the number of orifices.

\section{Application to encapsulating microparticles and cells}

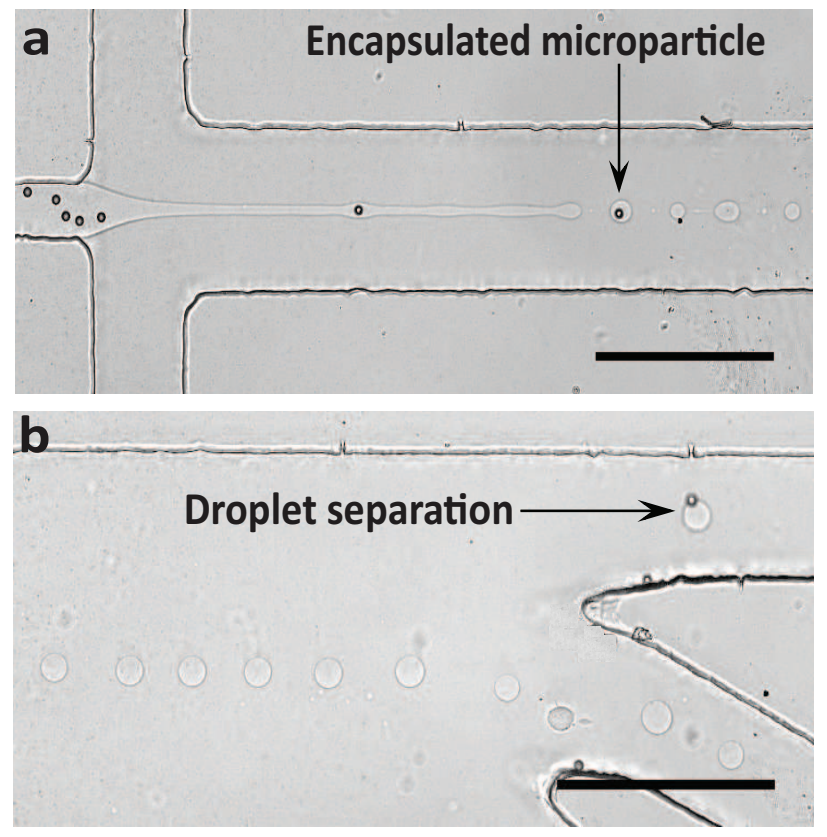

Figure 7: (a) An experimental image of the encapsulating of single microparticles. We use the ATPS 1 system, which has interfacial tension $\gamma=0.037 \mathrm{mN} / \mathrm{m}$. Microparticles suspended in the DEX phase are introduced at an inlet pressure $P_{D}=0.31 \mathrm{kPa}$. The PEG phase inlet pressure $P_{P}=0.40 \mathrm{kPa}$. As the DEX thread breaks into droplets, single microparticles are encapsulated inside the drops. (b) An image showing the separation of magnetic microparticle encapsulating ATPS droplets. Here, a magnetic gradient is generated by a permanent magnet that is placed adjacent to the microchannel. ATPS droplets that encapsulate magnetic microparticles are pulled by the magnetic microparticle to the side outlet. All other ATPS drops exit via the main outlet. Scale bars $200 \mu \mathrm{m}$. 
Some of the important applications of droplet microfluidics include single cell analysis, ${ }^{28,29}$ cell-based assays, ${ }^{30}$ and drug discovery. ${ }^{31}$ All of these applications require the encapsulation of biological materials inside aqueous droplets. Previous droplet microfluidics research have demonstrated the utility of encapsulating cells in droplets. In those systems, cells are encapsulated in aqueous droplets, which are then surrounded by a continuous oil phase. ${ }^{32-35}$ The oil phase is not biocompatible, so a cumbersome washing routine is required to isolate the cell encapsulating droplets, and then re-suspend them in a biocompatible aqueous phase.

In our approach, both disperse and continuous phases are aqueous and biocompatible. Therefore, cell encapsulating droplets could be used without washing and re-suspension in a different continuous phase.

We suspend $10 \mu \mathrm{m}$ diameter paramagnetic micrparticles in the ATPS 1 DEX phase, and encapsulate individual particles via our passive ATPS droplet scheme (Fig. 7 (a)). Individual microparticles enter the flow focusing junction, and are advected with the thin DEX phase thread. When the DEX phase thread breaks up into droplets, the individual microparticles become encapsulated inside DEX phase droplets (see also Supplementary Information Movie 4). Here, the DEX phase microparticle suspension is introduced at an inlet pressure $P_{D}=$ $0.31 \mathrm{kPa}$, and the PEG phase inlet pressure $P_{P}=0.40 \mathrm{kPa}$.

Since the microparticles are paramagnetic, we are also able to apply a magnetic field gradient, by placing a permanent magnet adjacent to the microchannel, to separate microparticle encapsulating ATPS drops from empty drops (Fig. 7 (b)). Here, the microparticle encapsulating drops are magnetically forced to a side outlet, while all of the empty ATPS drops flow into the main outlet.

To demonstrate the utility of our ATPS droplet generation scheme for cell encapsulation, we suspend PBMCs in the DEX phase, and use our ATPS droplet generation device to encapsulate the PBMCs (Fig. 8). The PBMCs are approximately $10 \mu \mathrm{m}$ in diameter. These results suggest that our completely passive, microfluidic flow focusing droplet generating 


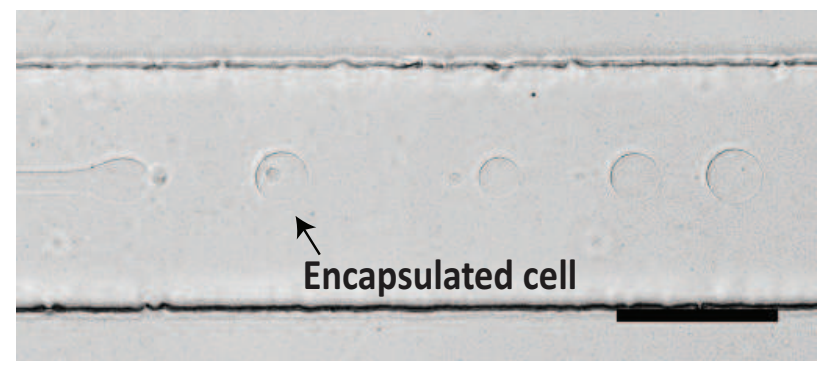

Figure 8: Proof-of-concept image of single cell encapsulation. Here, a PBMC is encapsulated in a DEX droplet that is surrounded by the PEG continuous phase. Scale bar $100 \mu \mathrm{m}$.

technique, may be used in the future to encapsulate single cells for biological applications such as cell screening, cell-based assays, and drug discovery - in a system that has biocompatibility in both the discrete and continuous phases.

\section{Conclusions}

In this manuscript, we describe a passive microfluidic flow focusing method to generate waterin-water ATPS droplets. Importantly, our ATPS droplet formation setup does not require any perturbations from external components, to generate the droplets. DEX and PEG phase fluids are introduced into the microfluidic device via simply controlled hydrostatic pressures, by attaching fluid-filled pipette tips to the device. ATPS droplets are generated passively by the Rayleigh-Plateau breakup of the DEX disperse phase thread.

We find that ATPS droplets generated in the jetting regime have a drop size CV of about $10 \%$. We also find highly monodisperse droplet formation, with the drop size CV less than $1 \%$, when the droplets form by dripping at the flow focusing junction.

We observe that the ATPS droplet radius scales with a power-law of the ratio of disperse and continuous phase inlet stresses, $a / h \propto\left(\frac{P_{D}}{\mu_{D}} / \frac{P_{P}}{\mu_{P}}\right)^{2}$.

To scale-up the production rate of ATPS droplets, we developed a multiplexed droplet formation setup, and show that it can produce up to $50+$ drops/sec with four parallel droplet generating orifices.

Finally, we show a proof-of-concept demonstration of microparticle and cell encapsula- 
tion, by encapsulating both paramagnetic microparticles and PBMCs. The microparticle encapsulating ATPS drops can be manipulated via application of a magnetic field gradient, to separate the encapsulating droplets from empty droplets. Our cell encapsulation experiments suggest that this technique may be applied in the future to encapsulate single cells in an all-biocompatible environment.

To the best of our knowledge, this microfluidic ATPS droplet generating method is the first that is completely passive. ${ }^{8,19}$ We anticipate that this system will find utility in many applications, especially because our ATPS droplet microfluidic system provides an improved biological environment compared to classical water-oil droplet microfluidic setups. ${ }^{36}$ For example, we anticipate that our technique may lead to improved microfluidic single cell analysis, ${ }^{37}$ production of functional microparticles, ${ }^{38}$ and controlled drug encapsulation and release. ${ }^{39}$

\section{Supporting information}

The Supporting Information is available free of charge on the ACS Publications website: Figures S1-3 and Movies 1-4.

\section{Acknowledgement}

S. S. H. Tsai (grant no. 435514-2013) and D. K. Hwang (grant no. 386092-2010) both acknowledge funding support from the Natural Sciences and Engineering Research Council (NSERC) Discovery grants program. The authors are grateful for PBMCs received as inkind contributions from STEMCELL Technologies Inc., Vancouver, Canada. The authors also thank E. S. L. Berndl for guidance in cell culturing, and M. C. Kolios for providing the cell culturing facilities. 


\section{References}

(1) Walter, H.; Johansson, G. Methods in Enzymology; Academic Press New York, 1994; Vol. 228.

(2) Hahn, T.; Hardt, S. Analytical Chemistry 2011, 83, 5476-5479.

(3) Münchow, G.; Hardt, S.; Kutter, J. P.; Drese, K. S. Lab on a Chip 2007, 7, 98-102.

(4) SooHoo, J. R.; Walker, G. M. Biomedical Microdevices 2009, 11, 323-329.

(5) Hatti-Kaul, R. Molecular Biotechnology 2001, 19, 269-277.

(6) Anna, S. L.; Mayer, H. C. Physics of Fluids 2006, 18, 121512.

(7) Seo, M.; Paquet, C.; Nie, Z.; Xu, S.; Kumacheva, E. Soft Matter 2007, 3, 986-992.

(8) Geschiere, S. D.; Ziemecka, I.; van Steijn, V.; Koper, G. J. M.; van Esch, J. H.; Kreutzer, M. T. Biomicrofluidics 2012, 6, 022007.

(9) Hardt, S.; Hahn, T. Lab on a Chip 2012, 12, 434-442.

(10) Song, Y. S.; Choi, Y. H.; Kim, D. H. Journal of Chromatography A 2007, 1162, 180186.

(11) Choi, Y. H.; Song, Y. S.; Kim, D. H. Journal of Chromatography A 2010, 1217, 37233728.

(12) Ziemecka, I.; van Steijn, V.; Koper, G. J.; Kreutzer, M. T.; van Esch, J. H. Soft Matter 2011, 7, 9878-9880.

(13) Ziemecka, I.; van Steijn, V.; Koper, G. J.; Rosso, M.; Brizard, A. M.; van Esch, J. H.; Kreutzer, M. T. Lab on a Chip 2011, 11, 620-624.

(14) Lai, D.; Frampton, J. P.; Sriram, H.; Takayama, S. Lab on a Chip 2011, 11, 3551-3554. 
(15) Shum, H. C.; Varnell, J.; Weitz, D. A. Biomicrofluidics 2012, 6, 012808.

(16) Sauret, A.; Shum, H. C. Applied Physics Letters 2012, 100, 154106.

(17) Song, Y.; Shum, H. C. Langmuir 2012, 28, 12054-12059.

(18) Moon, B.-U.; Jones, S. G.; Hwang, D. K.; Tsai, S. S. H. Lab on a Chip 2015, 15, 2437-2444.

(19) Song, Y.; Sauret, A.; Shum, H. C. Biomicrofluidics 2013, 7, 061301.

(20) Atefi, E.; Mann Jr, J. A.; Tavana, H. Langmuir 2014, 30, 9691-9699.

(21) Xia, Y.; Whitesides, G. M. Annual Review of Materials Science 1998, 28, 153-184.

(22) Guillot, P.; Colin, A.; Utada, A. S.; Ajdari, A. Physical Review Letters 2007, 99, 104502.

(23) Li, Z.; Mak, S. Y.; Sauret, A.; Shum, H. C. Lab on a Chip 2014, 14, 744-749.

(24) Ward, T.; Faivre, M.; Abkarian, M.; Stone, H. A. Electrophoresis 2005, 26, 3716-3724.

(25) Link, D. R.; Anna, S. L.; Weitz, D. A.; Stone, H. A. Physical Review Letters 2004, 92, 054503.

(26) Umbanhowar, P. B.; Prasad, V.; Weitz, D. A. Langmuir 2000, 16, 347-351.

(27) Nunes, J. K.; Tsai, S. S. H.; Wan, J.; Stone, H. A. Journal of Physics D: Applied Physics 2013, 46, 114002.

(28) Brouzes, E.; Medkova, M.; Savenelli, N.; Marran, D.; Twardowski, M.; Hutchison, J. B.; Rothberg, J. M.; Link, D. R.; Perrimon, N.; Samuels, M. L. Proceedings of the National Academy of Sciences 2009, 106, 14195-14200.

(29) Qi, D.; Hoelzle, D. J.; Rowat, A. C. The European Physical Journal Special Topics 2012, 204, 85-101. 
(30) Huebner, A.; Bratton, D.; Whyte, G.; Yang, M.; deMello, A. J.; Abell, C.; Hollfelder, F. Lab on a Chip 2009, 9, 692-698.

(31) Dressler, O. J.; Maceiczyk, R. M.; Chang, S.-I.; deMello, A. J. Journal of Biomolecular Screening 2014, 19, 483-496.

(32) Köster, S.; Angile, F. E.; Duan, H.; Agresti, J. J.; Wintner, A.; Schmitz, C.; Rowat, A. C.; Merten, C. A.; Pisignano, D.; Griffiths, A. D.; Weitz, D. A. Lab on a Chip 2008, 8, 1110-1115.

(33) Yu, L.; Chen, M. C.; Cheung, K. C. Lab on a Chip 2010, 10, 2424-2432.

(34) Clausell-Tormos, J.; Lieber, D.; Baret, J.-C.; El-Harrak, A.; Miller, O. J.; Frenz, L.; Blouwolff, J.; Humphry, K. J.; Köster, S.; Duan, H.; Holtze, C.; Weitz, D. A.; Griffiths, A. D.; Merten, C. A. Chemistry $\&$ Biology 2008, 15, 427-437.

(35) Chabert, M.; Viovy, J.-L. Proceedings of the National Academy of Sciences 2008, 105, $3191-3196$.

(36) Keating, C. D. Accounts of Chemical Research 2012, 45, 2114-2124.

(37) Mazutis, L.; Gilbert, J.; Ung, W. L.; Weitz, D. A.; Griffiths, A. D.; Heyman, J. A. Nature Protocols 2013, 8, 870-891.

(38) Yuet, K. P.; Hwang, D. K.; Haghgooie, R.; Doyle, P. S. Langmuir 2009, 26, 4281-4287.

(39) Duncanson, W. J.; Lin, T.; Abate, A. R.; Seiffert, S.; Shah, R. K.; Weitz, D. A. Lab on a Chip 2012, 12, 2135-2145. 
Graphical TOC Entry

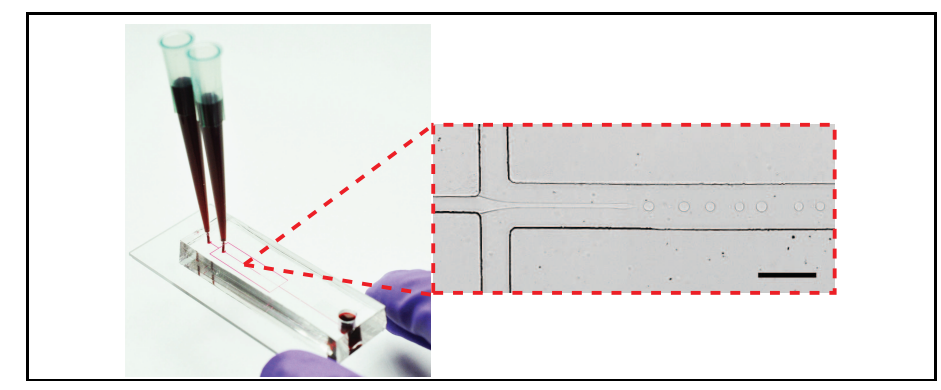

